\title{
Hard X-ray micro-tomography of a human head post-mortem as a gold standard to compare $\mathrm{X}$-ray modalities
}

\author{
M. Dalstra*a,b, G. Schulzc , D. Dagassan-Berndt ${ }^{\mathrm{d}}$, C. Verna ${ }^{\mathrm{a}}$, M. Müller-Gerble, B. Müller ${ }^{\mathrm{c}}$ \\ ${ }^{a}$ Department of Orthodontics and Pediatric Dentistry, University Center for Dental Medicine, \\ Hebelstrasse 3, 4056 Basel, Switzerland; 'bection of Orthodontics, Institute of Odontology, Aarhus \\ University, Vennelyst Boulevard 9, 8000 Aarhus C, Denmark; 'Biomaterials Science Center (BMC), \\ University of Basel, Gewerbestrasse 14, 4123 Allschwil, Switzerland; ${ }^{\mathrm{d} D e p a r t m e n t ~ o f ~ O r a l ~ S u r g e r y, ~}$ \\ Oral Radiology and Oral Medicine, University Center for Dental Medicine, Hebelstrasse 3, 4056

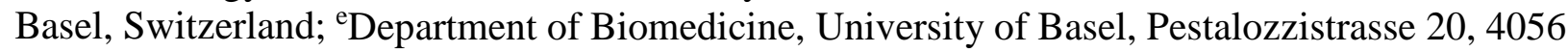 \\ Basel, Switzerland
}

*michel.dalstra@unibas.ch; phone 45 60684190; fax 61267 25 81; odont.au.dk/om-odontologi/sektioner/or/

\begin{abstract}
An entire human head obtained at autopsy was micro-CT scanned in a nano/micro-CT scanner in a 6-hour long session. Despite the size of the head, it could still be scanned with a pixel size of $70 \mu \mathrm{m}$. The aim of this study was to obtain an optimal quality 3D data-set to be used as baseline control in a larger study comparing the image quality of various cone beam CT systems currently used in dentistry.

The image quality of the micro-CT scans was indeed better than the ones of the clinical imaging modalities, both with regard to noise and streak artifacts due to metal dental implants. Bony features in the jaws, like the trabecular architecture and the thin wall of the alveolar bone were clearly visible. Therefore, the 3D micro-CT data-set can be used as the gold standard for linear, angular, and volumetric measurements of anatomical features in and around the oral cavity when comparing clinical imaging modalities.
\end{abstract}

Keywords: X-ray tomography, micro-CT, cone beam CT, bone, dental tissues

\section{INTRODUCTION}

Cone beam CT (CBCT) scanning has gained much popularity since its introduction in the field of dentistry in $2003^{1}$. Especially in the specialties of oral and maxillofacial surgery, dental implantology and orthodontics, CBCT has proved its worth as a tool for diagnosis and treatment planning. With an effective radiation dose being up to almost an order of magnitude lower than a comparable scan with conventional $\mathrm{CT}^{2}$, it is a very good option for scanning the head region, especially with young patients, like often the case in orthodontics. However, the price to pay for this reduced radiation is the lower depth in gray values in the images. Although the difference between hard and soft tissues can be easily be distinguished, differences within these tissues, like different muscles, are in general more difficult, if not impossible, to discern. Variations in the scanning settings, in particular the choice of field-of-view and the exposure settings, will affect the image quality ${ }^{3}$. Due to its popularity, many manufacturers of radiological equipment have started to produce CBCT systems and more than two dozen different systems are on the market at present ${ }^{4}$. With different hardware components, like X-ray sources and detectors, used by the different manufacturers for their systems further variations in image quality are introduced ${ }^{4}$. The local tomography at the selected scan settings makes the data interpretation therefore often difficult $^{5}$. For this reason, it was decided to perform a tomography study of the same human donor head with multiple CBCT scanners, each time scanned with a variety of machine settings. Comparisons of different CBCT systems have been made before ${ }^{2,6,7}$, but with a phantom or a dry skull; never using an entire human head. As it was a unique chance to be able to scan this human head, it was decided to include other scanning modalities in this comparison as well, including multi-slice CT, MRI and micro-CT. Although the latter methodology for obvious reasons cannot be used in clinical practice, the image quality can be expected to be superior to all other scanning modalities and therefore the scans

Developments in X-Ray Tomography X, edited by Stuart R. Stock, Bert Müller, Ge Wang, Proc. of SPIE Vol. 9967, 99670P · (c) 2016 SPIE · CCC code: 0277-786X/16/\$18 · doi: 10.1117/12.2237655 
obtained with micro-CT were meant to be a gold standard for the image quality of the other modalities. Occasionally, micro-CT has been compared to CBCT before, but only using small-sized samples, like bone biopsies ${ }^{8}$ or individual teeth $^{9}$. The challenge of the present study is to scan an entire human head, while maintaining the superior image quality of micro-CT.

\section{MATERIALS AND METHODS}

\subsection{Material}

The human head from an elderly male donor was obtained at autopsy and preserved at the Department of Anatomy of the University of Basel. Permission for the use of the head for this study was provided by the local ethics committee. The head was kept in a series of plastic bags at all times to keep it moist and for the ease of transport and handling. A number of teeth in the maxilla had restorations in the form of root canal fillings, amalgam fillings and gold crowns. As the dental records of the donor were not available, the exact material composition of these restorations could not be traced.

\subsection{Cone beam computerized tomography}

Prior to micro-CT, the head had been scanned at multiple occasions with various CBCT systems, each with a number of machine settings, at different locations in Switzerland and southern Germany. In addition, also conventional CT and MRI were performed at Basel University Hospital. A total of 78 data-sets were collected thus. As the prime question for the comparison of these different data-sets stemmed from an orthodontic angle, the main variables for comparison were those, which are important in orthodontic treatment planning: the quality of the alveolar bone and the thickness of the alveolar wall. The results of these comparisons are the subject of an ongoing study to be published at a later point in time.

\subsection{Micro-tomography}

Micro-CT of the head was performed at the Biomaterials Science Center of the University of Basel. It was placed in a dedicated sample holder and fixed on the rotation stage of a nanotom ${ }^{\circledR} \mathrm{m}$ scanner (Phoenix|X-ray, GE Sensing \& Inspection Technologies GmbH, Wunstorf, Germany). The device is equipped with a $180 \mathrm{kV} / 15 \mathrm{~W}$ nanofocus X-ray source and a temperature stabilized digital GE DXR detector $(3,072 \times 2,400$ pixels $)$ with a pixel length of $100 \mu \mathrm{m}$. With a source-specimen distance of $400.0 \mathrm{~mm}$ and a source-detector distance of $571.4 \mathrm{~mm}$, the magnification factor was 1.4285 , which resulted in a pixel length of $70 \mu \mathrm{m}$. For the high absorbing specimen, the maximal acceleration voltage of $180 \mathrm{kV}$ and a beam current of $30 \mu \mathrm{A}$ were adjusted and the mean photon energy was increased by adding a $0.5 \mathrm{~mm} \mathrm{Cu}$ filter between the source and the specimen. 1,800 equiangular radiographs were taken over $360^{\circ}$. At each rotation angle three averages with an exposure time of $3 \mathrm{~s}$ each were acquired. The whole scanning procedure took about 6 hours.

After reconstruction using a cone beam filtered back-projection algorithm, the resulted 3D data-set had an isotropic voxel size of $70 \mu \mathrm{m}$. In order to reduce the size of the scans for the ease of handling, the data-set was binned with a factor 2, yielding an effective voxel size of $140 \mu \mathrm{m}$. The single-slice images were then exported in DICOM format for further inspection and analysis. The visualization of the data-set was performed using both VGStudio MAX 2.1 (Volume Graphics GmbH, Heidelberg, Germany) and Mimics, ver. 18 (Materialise, Leuven, Belgium) imaging softwares.

\section{RESULTS}

An overview image of the 3D data-set is shown in Figure 1. Some moderate streak artifacts due to the metal restorations can be observed in the upper jaw. By trying to exclude these artifacts when adapting the histogram of the gray values in the segmentation of the data-set for 3D reconstruction of the hard tissues, the side segments of the dental arch as well as their surrounding bony structures tend to disappear (Fig. 1, bottom right). Otherwise, the detailed images show a crisp trabecular structure of the cancellous bone inside the maxilla and mandible with the individual trabeculae clearly visible (Figs. 2 and 3). Also the thickness of the alveolar wall around the roots of the teeth can clearly be discerned, at least for those teeth without metal reconstructions. 
As a comparison to the micro-CT images, a selection of different images from the same mandible taken with three CBCT systems and a multislice CT is shown in Figure 4. At the same magnification, the images appear generally more blurred.

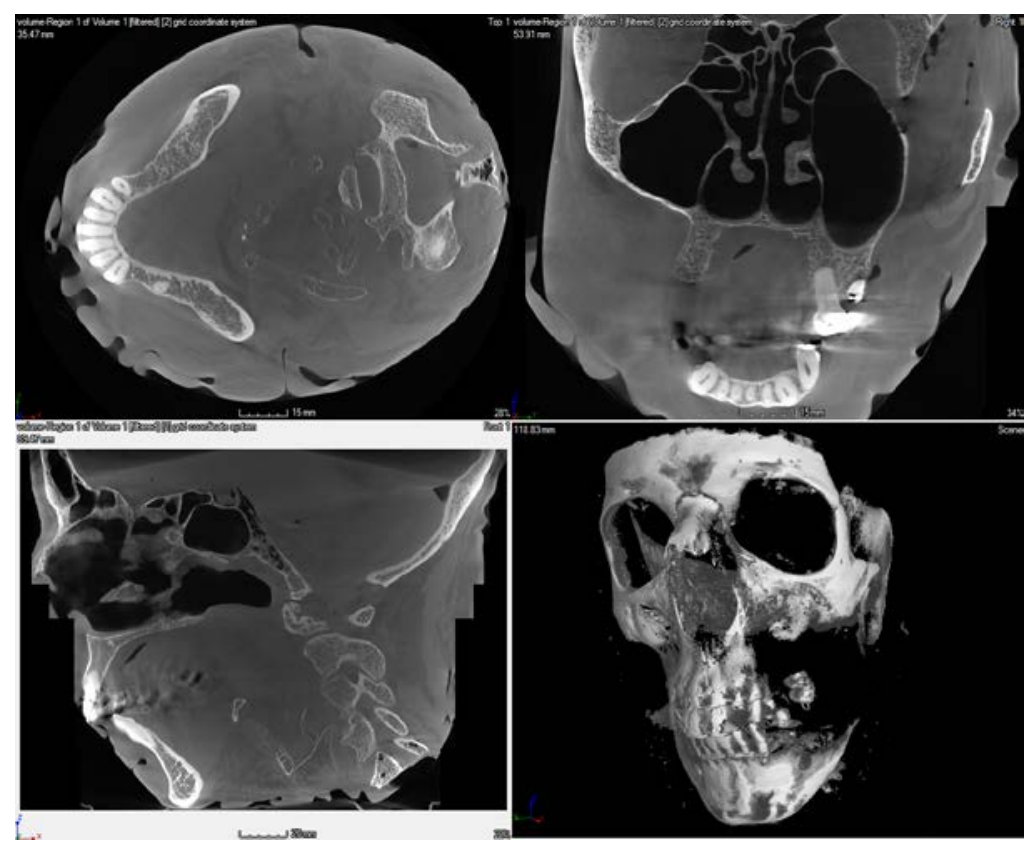

Figure 1. Three orthogonal cross-sectional views, coronal (top left), frontal (top right) and sagittal (bottom left), and a $3 \mathrm{D}$ reconstruction (bottom right) of the entire data-set.

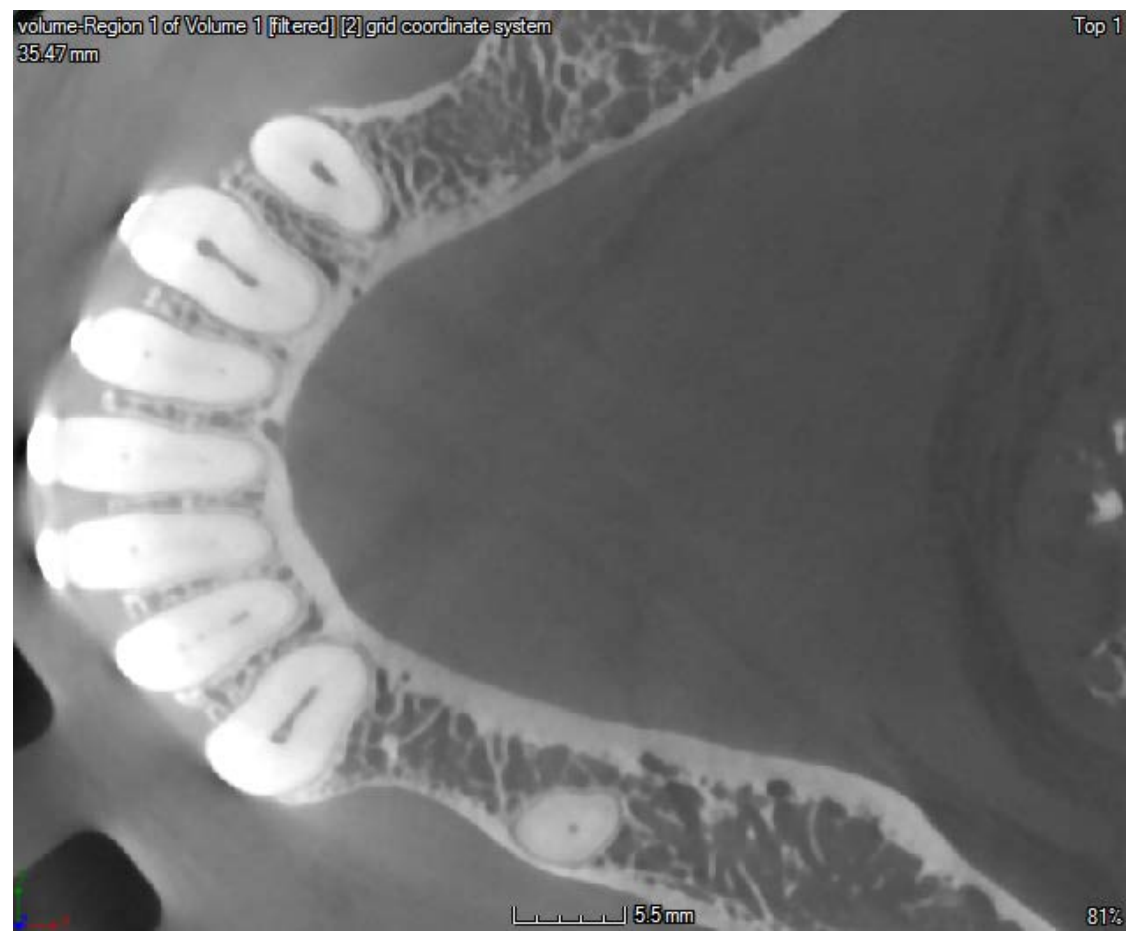

Figure 2. Detailed view from a coronal section through the mandible. Note the trabecular structure of the alveolar bone. 


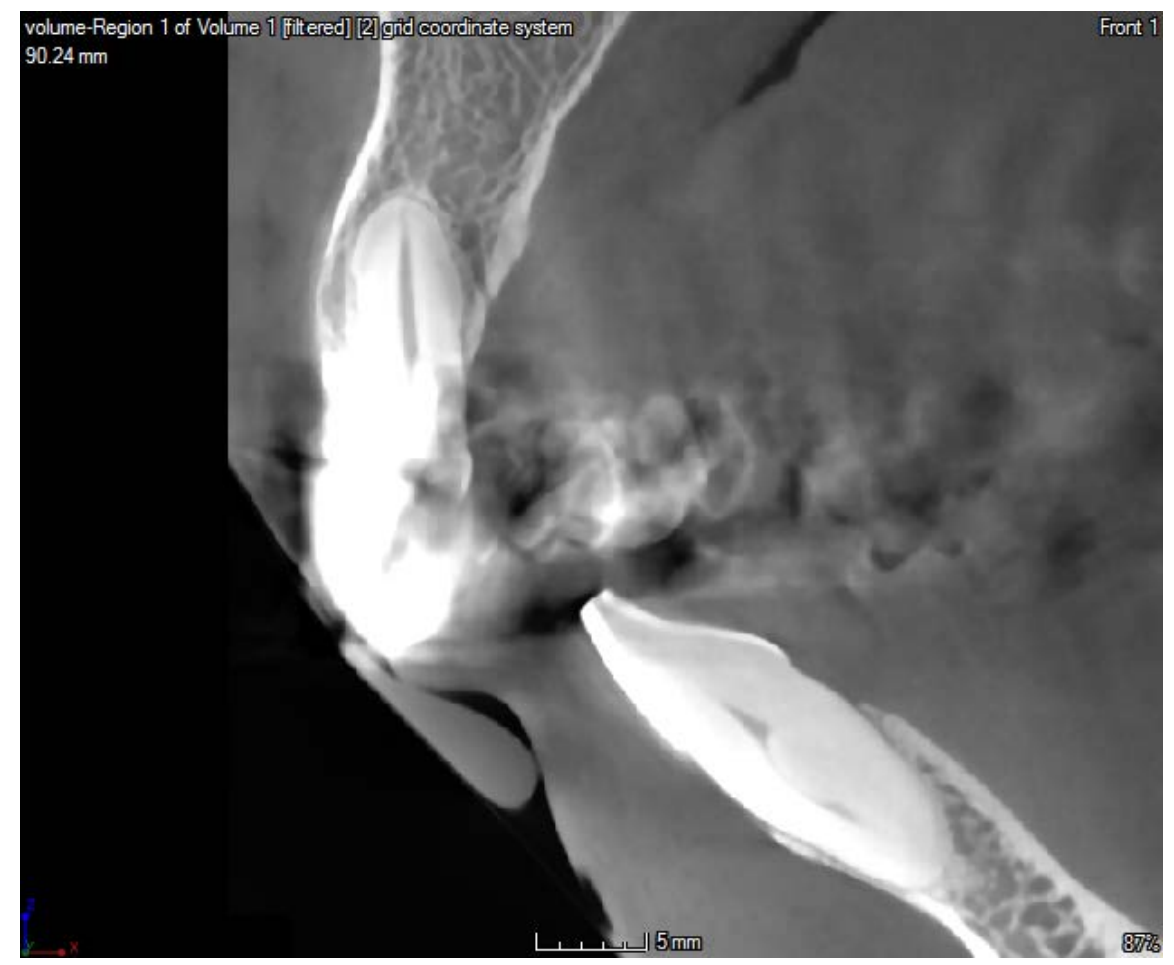

Figure 3. Detailed view from a sagittal section through the oral cavity. Note the trabecular structure of the alveolar bone in the mandible and maxilla. Also note the thickness of the alveolar wall of the mandible.

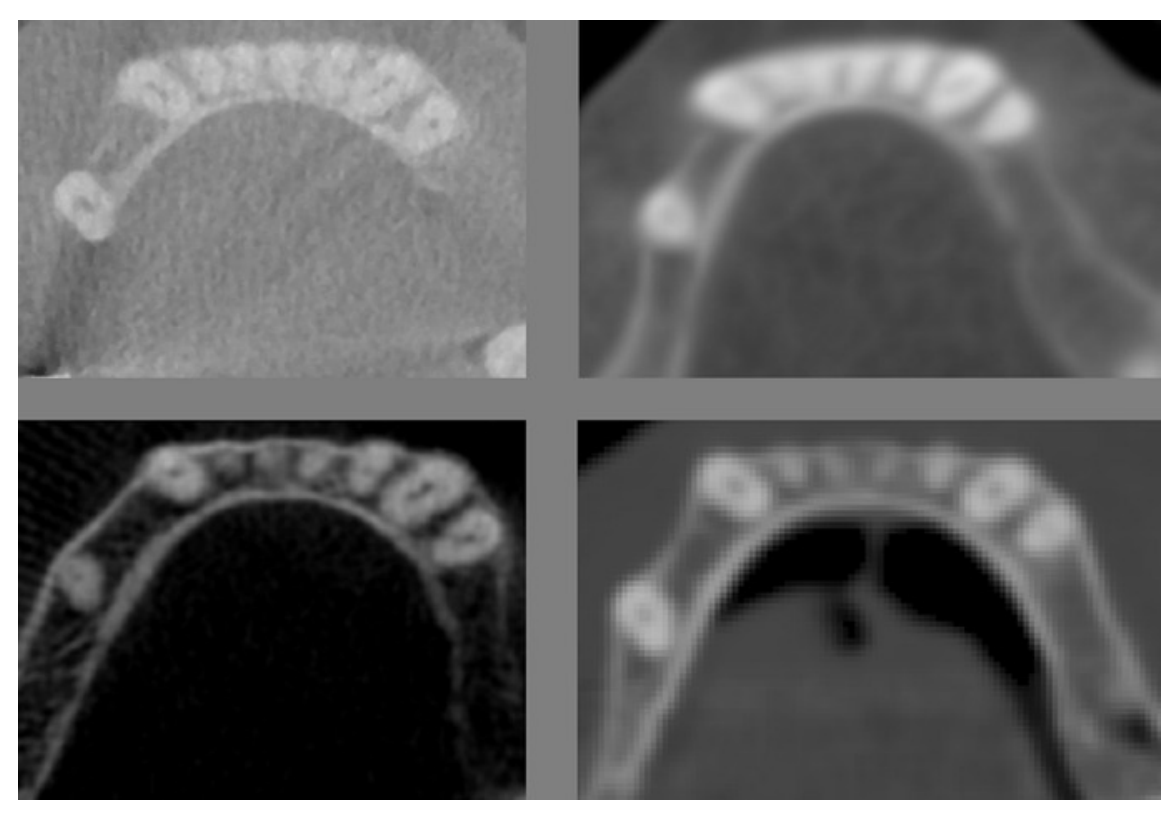

Figure 4. Detailed views from coronal sections from data-sets obtained with the 3D Accuitomo (J. Morita MFG. Corp., Kyoto, Japan, top left), Promax 3D (Planmeca OY, Helsinki, Finland, top right), Scanora 3Dx (Soredex, Tuusula, Finland, bottom left) and Emotion 16 (Siemens Healthcare GmbH, Erlangen, Germany, bottom right). CT image voxel sizes are $0.25,0.60,0.30$ and $0.47 \mathrm{~mm}$, respectively. 


\section{DISCUSSION}

A micro-CT of an entire human head has been performed. Due to the size of the head, the best achievable resolution was a pixel size of $70 \mu \mathrm{m}$, which is still almost a factor 4 better than the smallest pixel size obtainable with CBCT. For the purpose of comparing the micro-CT scans to the images from the clinical scanning modalities, particular attention was paid to the quality of the alveolar bone and the thickness of the alveolar wall around the dental roots. Most CBCT scans do not depict the quality of the alveolar bone well. It is very difficult to discern any trabecular bone between the roots (Fig. 4). As most trabeculae are smaller than the pixel size, the gray values of the pixels only represent an average tissue density. On the other hand in the micro-CT scans the individual trabeculae are clearly visible, as well as the thin lamina dura surrounding the roots and even the space of the periodontal ligament in between the lamina dura and the root (Figs. 2 and 3) is still distinguishable. The thickness of the alveolar bone around the roots, especially in the bucco-lingual direction is a variable, which is easier quantifiable than the alveolar bone quality. In the micro-CT scans, this thickness is well defined with a sharp border between the bone and the surrounding soft tissues and air pockets. Although the alveolar wall is also visible in the clinical scanning modalities, it does not appear with the same sharpness as in the micro-CT scans. The border appears more blurry, which can be attributed to partial volume effects in the pixels located in the border area. This blurriness is largely dependent on the machine settings and type of scanner. It will make the measurement of the thickness of the wall more prone to inaccuracies as the choice for picking the points from where to where to measure are more dependent of the subjectivity of the observer. In particular overestimation of the actual thickness can be a danger here, which may potentially have dire consequences for orthodontic treatment planning. The presence of streak artifacts due to the presence of metal restorations can be a problem when assessing the alveolar bone in the vicinity, but in the micro-CT data-set these artifacts are not as extensive as in the clinical scanning modalities. They do affect the 3D reconstruction, however, when employing a global threshold segmentation routine ${ }^{10}$. The regions with thin and/or less dense bone tend to disappear (Fig. 1). In these cases, local or adaptive thresholding segmentation or other metal artifact reduction algorithms might be used instead ${ }^{11}$. However, the original purpose of the study to obtain a 3D data-set using hard X-ray tomography as a gold standard for clinical 3D imaging modalities was accomplished.

\section{CONCLUSION}

As expected, the image quality of the nanotom ${ }^{\circledR} \mathrm{m}$ data-set was better than the ones obtained with the clinical 3D imaging modalities, both with regard to noise and streak artifacts due to metal dental implants and the effects of beam hardening artifacts due to the larger pixel sizes of the clinical scanning modalities. With the appearance of the alveolar bone qualitatively and quantitatively assessable, the 3D micro-CT data-set can be used as a gold standard for linear, angular, and volumetric measurements of anatomical features in and around the oral cavity when comparing clinical imaging modalities.

\section{REFERENCES}

[1] Ludlow, J.B., Davies-Ludlow, L.E., Brooks, S.L., "Dosimetry of two extraoral direct digital imaging devices: NewTom cone beam CT and Orthophos Plus DS panoramic unit," Dentomaxillofac Radiol 32, 229-234 (2003).

[2] Suomalainen, A., Kiljunen, T., Käser, Y., Kortesniemi, M., "Dosimetry and image quality of four dental cone beam computed tomography scanners compared with multislice computed tomography scanners," Dentomaxillofac Radiol 38, 367-378 (2009).

[3] Scarfe, W.C., Li, Z., Aboelmaaty, W., Scott, S.A., Farman, A.G., "Maxillofacial cone beam computed tomography: essence, elements and steps to interpretation," Austral Dent J 57 (1 Suppl), 46-60 (2012).

[4] Kiljunen, T., Kaasalainen, T., Suomalainen, A., Kortesniemi, M., "Dental cone beam CT: A review," Phys Med 31, 844-860 (2015). 
[5] Dillenseger, J.P., Gros, C.I., Sayeh, A., Rasamimanana, J., Lawniczak, F., Leminor, J.M., Matern, J.F., Constantinesco, A., Bornert, F., Choquet, P., "Image quality evaluation of small FOV and large FOV CBCT devices for oral and maxillofacial radiology," Dentomaxillofac Radiol (2016), Epub ahead of print.

[6] Alquerban, A., Jacobs, R., Fieuws, S., Nackaerts, O., The SEDENTEXCT Project Consortium, Willems, G. "Comparison of 6 cone-beam computed tomography systems for image quality and detection of simulated canine impaction-induced external root resorption in maxillary lateral incisors," Am J Orthod Dentofacial Orthop 140, e129-e139 (2011)

[7] Esmaeli, F., Johari, M., Haddadi, P., Vatankhah, M. "Beam hardening artifacts: Comparison between two cone beam computed tomography scanners," J Dent Res Dent Clin Prospect 6, 49-53 (2012).

[8] Monje, A., Monje, F., González-Garciá, R., Galindo-Moreno, P., Rodriguez-Salvanes, F., Wang, H.Y., "Comparison between microcomputed tomography and cone-beam computed tomography radiologic bone to assess atrophic posterior maxilla density and microarchitecture," Clin Oral Impl Res 25, 723-728 (2014).

[9] Maret, D., Peters, O.A., Galibourg, A., Dumoncel, J., Esclassan, R., Kahn, J.L., Sixou, M., Telmon, N., "Comparison of the accuracy of 3-dimensional cone-beam computed tomography and micro-computed tomography reconstructions by using different voxel sizes," J Endod 40, 1321-1326 (2014).

[10]Hassan, B., Souza, P.C., Jacobs, R., de Azambuja Berti, S., van der Stelt, P., "Influence of scanning and reconstruction parameters on quality of three-dimensional surface models of the dental arches from cone beam computed tomography," Clin Oral Invest 14, 303-310 (2010).

[11] Meyer, E., Raupach, R., Lell, M., Schmidt, B., Kachelriess, M., "Normalized metal artifact reduction (NMAR) in computed tomography," Med Phys 37, 5482-5493 (2010). 